\title{
Nurses' professional values and its Relation to their Burnout in Minia university hospitals
}

\author{
Eman Mahmoud Mohamed ${ }^{1}$, Safaa Mohammed Abdelrahman ${ }^{2}$, Mona Thabet $^{3}$
}

1. (B.Sc. Nursing);

2. Professor of Nursing Administration, Faculty of Nursing, Minia University,

3. Lecturer in Nursing Administration Department, Faculty of Nursing, Minia University

\begin{abstract}
Background: An awareness of professional values, cultural differences, and bases help to avoid ineffective communication in stressful situations which can help staff nurses in preventing their feeling of burnout. Aim: To assess nurses' professional values and its relation to their Burnout in Minia university hospitals. Research design: A descriptive cross- sectional research design. Subjects: A representative sample $30 \%$ from total staff nurses who working in Minia University Hospitals $(\mathrm{N}=337)$. Setting: The study was conducted in Minia University Hospitals, Egypt. Tools of data collection: included two tools; Nurses Professional Values Scale (NPVS), and Maslach's Burnout Inventory scale. Results: Staff nurses had moderate mean score level for all subscale of professional values; also there were more than three quarter $(78.3 \%)$ of the staff nurses had moderate score level of total professional value, less than one quarter $(20.8 \%)$ of them had high score level degree of total professional value. Also, there were less than two third (62.9\%) of staff nurses had high total score levels of Maslach's burnout inventory; more than one third (37.1\%) of them have moderate score level; and no one of them had low burnout level score. Conclusion: The findings of this study revealed that staff nurses had moderate to high level of professional values perception as well as their burnout level. Also, there were negative correlation between professional values and level of burnout. Recommendation: Training programs should be designed continuously for staff nurses to improve their professional values perception and decrease their burnout level.
\end{abstract}

Keywords: Burnout, Nurses, Minia university hospitals, Relation, Professional values.

\section{Introduction:}

Society has evolved the nursing profession to meet the health needs, and it is a specialized health practice that deals with individual, family and community health. Thus, nurses should hold professional values which underlie their moral and ethical responsibilities towards their patients, communities, careers, employers and themselves (Ayla, et al., 2018). Also, the quality and the delivery of health care effectiveness have been affected by the roles of nurse; so they should continuous developing their career (Abo Elmaged\& Ahmed, 2019).

Professional values are the criteria for behavior that is rooted in personal values, which are influenced by family, culture, environment, religion, and ethnicity. Also, the process of values acquisition is gradual and evolutionary; and occurs throughout an individual's lifetime. Professional values are a source to promote nurses' ethical competencies in clinical settings and dealing with ethical concerns in the present era (Kim et al., 2015).

Most nurses expected to be aware of professional values and apply them to their decision makings while dealing with such ethical problems, development of professional values in nurses is mainly influenced by experts in the profession, colleagues, patient care situations, and organizational values (Poorchangizi, et al., 2019).

Nurses' professional values are important because they affect the decisions made in caring for patients as well as nurses burnout. Nurses need to be aware of their professional values and at the same time be able to care for patients who have different values. An awareness of professional values, cultural differences, and bases help to avoid ineffective communication in stressful situations. Preventing burnout requires many of the same coping skills that we use to combat reactions to any stressor and reduce burnout (Elsheikh \& Naga, 2021).

P a g e | 106
Burnout is the state of mind developed among serviceoriented professionals. It can reduce effectiveness, as well as declining the motivation and engagements in non-workrelated behaviors. Burnout is defined as follows: "psychological syndrome that involves a prolonged response to chronic interpersonal stressors on the job." Burnout is a mental state developed over the period in a work environment with continuous stress related to work demands. If this mental-state is prolonged, it can cause physical and emotional distress (Long et al., 2014)

Burnout has three dimensions termed as the burnout inventory; namely: emotional exhaustion, depersonalization, and reduction in personal accomplishment (WHO, 2019). The emotional exhaustion is the prime factor of the burnout and associated with the overextension of work-related efforts physically and emotionally and leads to the depletion of perceived personal resources (Kim\& Yoon; 2018). Depersonalization is the loss of feeling towards work-related issues and behaving in a non-human way. It moves an individual self away from the work-related issues and so they perform without empathy in the work setting (Heijden, et al., 2019).

When an individual experiences emotional exhaustion and depersonalization, they will lose the desire for achievement and competence to perform. So; burnout is the outcome of extended occupational stress that influences the individuals' service provider and service receiver in the health industry (Pérez-Fuentes, et al., 2019).

Therefore, Professional value and emotional exhaustion, depersonalization, and low personal accomplishment were all correlated. The professional value of nurses played an important role in the level of burnout. When nurses believe the job is important and valuable, they will feel satisfied and will seldom burnout. In contrast, when the job is 
not believed to be valuable, burnout will occur in daily routine work (Xie et al., 2021).

\section{Significance of the study:}

Professional nursing values affect the quality of nurses' professional life and play an important role in nurses' degree of burnout and their decision upon staying in or leaving their jobs or even the profession. In the case that nursing students or nurses rely on the values that conflict with those adopted by the profession, the profession's image and identity may be at risk (Posluszny\& Hawley, 2017).

Monroe, (2019) done study and measure the professional values of registered nurses and reported that higher score indicated stronger orientation toward professional nursing values and nurses with experience of 10 years or more had the highest scores. Also, Abbas et al. (2020) found that approximately one-quarter of nurses and physicians who working in ICUs in Canal health sector have high levels of burnout syndrome.

Study done by Abdalla et al., (2016) aimed to find out the relationship between professional values and burnout among nurses in Assuit University hospital in general and specialized medical, surgical and intensive care units, concluded that there was a positive correlation between nurses' professional values and burnout. So, examining the perspective of nurses on the relevance of professional values in various environments and cultures will allow healthcare administrators to interpret the gaps in the value systems of professional people, creating an acceptable working atmosphere for nurses

\section{Aim of the Study:}

The aim of the current study is to assess the professional values and its relation to burnout among nurses at Minia university hospitals.

\section{Research Questions:}

1. What is the level of professional values among nursing staff at Minia university hospitals?

2. What is the level of burnout among nursing staff at Minia university hospitals?

3. Is there a relation between professional values and burnout among nurses at Minia university hospitals?

\section{Subjects and methods \\ Research Design}

Descriptive cross-sectional research design was utilized to fulfill the aim of this study.

\section{Setting:}

The study was conducted in Minia University Hospitals, Minia City, Egypt which were Minia Emergency University Hospital; Minia Nephrology and Urology University Hospital; Mina Cardio Thoracic Surgery University hospital; and Minia Pediatric and Obstetrics university hospital

\section{Subjects}

The subjects of the study sample was included representative sample $(30.0 \%)$ which was selected randomly from all staff nurses who working in Minia University Hospitals during the period of data collection. Their total numbers was (1121) and study subject was (337).
Data Collection Tools:

Data was collected through the utilization of two tools as follows:

Tool (1): Nurses Professional Values Scale (NPVS)

It was included two parts:

\section{Part I: Personal data}

It was used to collect data about nurses and encompass items such as, age, gender, marital status, educational level, position, years of experience and department.

Part II: Nurses Professional Values Scale. It was adopted from American Nurse Association (ANA) code of ethics for nurses (1985) and was translated by the researcher. It was used to assess nurse professional value. It consisted of 44 items of expression point; and this part divided into eleven subscale as follows; Human dignity ( 5 items), Privacy (3 items), Safeguard client (4items), Responsibility, and accountability (4items), Competence (4items), Informed judgment (4items), Contributes to knowledge (4items), Improve standards (4items), Conditions of employment (4items), Maintain integrity (3 items), and Collaborate (5 items).

The items were measured by using five-points Likert scale ranged as $(5=$ most important, $4=$ very important, $3=$ important $2=$ somewhat important, and $1=$ not important). The scoring system of the tool was as follows:

- Low professional values score if the total scores ranged from (44- 102).

- Moderate professional values score if the total scores ranged from $(103-161)$

- High professional values score if the total scores ranged from (162- 220).

Tool (2): Maslach's Burnout Inventory scale: This tool developed by Maslach and Jackson, (1997). It was used to assess the degree of burnout among nurses; and consisted of (22) items. The items were grouped under three theoretical dimensions as emotional exhaustion (8 items), depersonalization ( 8 items), and personal achievement (6 items).

The items was measured by using seven-points Likert scale ranged as $0=$ never, $1=$ a few times a year or less, $2=$ once a month or less, $3=$ A few times a month, $4=$ once a week, $5=$ a few times a week, and $6=$ every day.

A high degree of burnout is reflected in high scores on the emotional exhaustion (maximum score of 54) and depersonalization subscales (maximum score of 30) and in low scores on the reduced professional accomplishment subscale (maximum score of 48). The scoring system was as follow:

- Low burnout level if the total scores ranged from (033)

- Moderate burnout level if the total scores ranged from (34- 66)

- Low burnout level if the total scores ranged from (67- 132)

Validity and Reliability of Tools: Validity of the tools:

The tools were tested for the content validity by a jury of five experts' in the field of nursing administration with no modification was done. 


\section{Reliability of the tools:}

The tools were tested for internal reliability by using Cronbach' alpha test, illustrated as follows thus indicating a high degree of reliability; it was $(\alpha=0.917)$ for Total Nurses Professional Values and it was $(\alpha=0801)$ for Total Maslach's Burnout Inventory.

\section{Pilot Study:}

A pilot study was carried out before starting data collection on $10 \%$ of nurses (34) from Minia University Hospitals. The aim of this pilot study was to test the clarity, comprehensiveness, accessibility, and applicability of the tools and to estimate the appropriate time require filling the questionnaire.

\section{Data Collection Procedure:}

Participants were recruiting from Minia University Hospitals. Each participant was receiving the questionnaires to fill them after taking their consent to participate in the study and given an explanation about the nature of the study by the researcher.

The tools were distributed to all the participants after explaining the purpose and process of data collection. Tools were directly administered and supervised by the researcher with the assistance of head nurse for each department through morning and evening shift during the working days.

Participants were given from 25 minute to 30 minutes to answer all the tools. They were allowed to discuss with researcher any item that needs more clarification. The data collection was performed from staff nurses, during the period of 4 months from the beginning of February 2021 to the beginning of June 2021.

\section{Ethical Consideration:}

- Approvals were granted from the Research Ethics Committee, and Dean of the Faculty of Nursing, Minia University

- Permissions were obtained from the directors of the Minia University Hospitals and the chief persons of the nursing department.

- Consent was obtained from nurses that were willing to participate in the study, after explaining the nature and purpose of the study. Study subject has the right to refuse to participate or withdraw from the study without any rational any time.

- Study subject privacy was considered; and they were assured that all their data are highly confidential; anonymity was also assured through assigning a number for each nurse instead of names to protect their privacy.

\section{Data statistical analysis}

Data entry was done using compatible personal computer. Statistical analysis done by using statistical package of social science (SPSS, IBM) version 25 and excel for figures. The content of each tool was analyzed, categorized, and then coded. Data were presented using descriptive statistics in the form of frequencies and percentages for qualitative variables and means and standard deviations for quantitative variables. Statistical significance used at $P$ value $<0.05$.

\section{Results}

Table (1): Distribution of the staff nurses according to their personal data $(n=337)$.

\begin{tabular}{|c|c|c|c|}
\hline \multirow{2}{*}{\multicolumn{2}{|c|}{ Personal data }} & \multicolumn{2}{|c|}{ Staff nurses $(n=337)$} \\
\hline & & No. & $\%$ \\
\hline \multicolumn{4}{|l|}{ Age / years } \\
\hline 0 & $18-<28$ & 265 & 78.6 \\
\hline$\bullet$ & $28-<38$ & 49 & 14.6 \\
\hline$\bullet$ & $38-<49$ & 23 & 6.8 \\
\hline \multicolumn{2}{|c|}{ Mean \pm SD } & \multicolumn{2}{|c|}{$26.5 \pm 6.1$ year } \\
\hline \multicolumn{4}{|l|}{ Marital status } \\
\hline$\bullet$ & Single & 146 & 43.3 \\
\hline$\bullet$ & Married & 191 & 56.7 \\
\hline \multicolumn{4}{|l|}{ Current position } \\
\hline$\bullet$ & Nurse & 170 & 50.4 \\
\hline$\bullet$ & Bedside nurse & 114 & 33.8 \\
\hline$\bullet$ & Head nurse & 53 & 15.8 \\
\hline \multicolumn{4}{|c|}{ Years of experience in the current department } \\
\hline$\bullet$ & $1-<6$ & 275 & 81.5 \\
\hline$\bullet$ & $6-<11$ & 34 & 10.1 \\
\hline$\bullet$ & $11-16$ & 10 & 3.0 \\
\hline$\bullet$ & $16-<21$ & 9 & 2.7 \\
\hline$\bullet$ & $21-25$ & 9 & 2.7 \\
\hline Mean \pm SD & & \multicolumn{2}{|c|}{$5.6 \pm 6.3$ year } \\
\hline
\end{tabular}

Table (1): presents that there are (78.6\%) of staff nurse's aged between $18-<28$ with mean age $26.5 \pm 6.1$ years; and there are (56.7) of them married. Regarding current position, there are (50.4\%) of them work as a nurse, and the majority $(81.5 \%)$ of them have from $1-<6$ years with mean experience $5.6 \pm 6.3$ years in the nursing field 


\section{Gender}

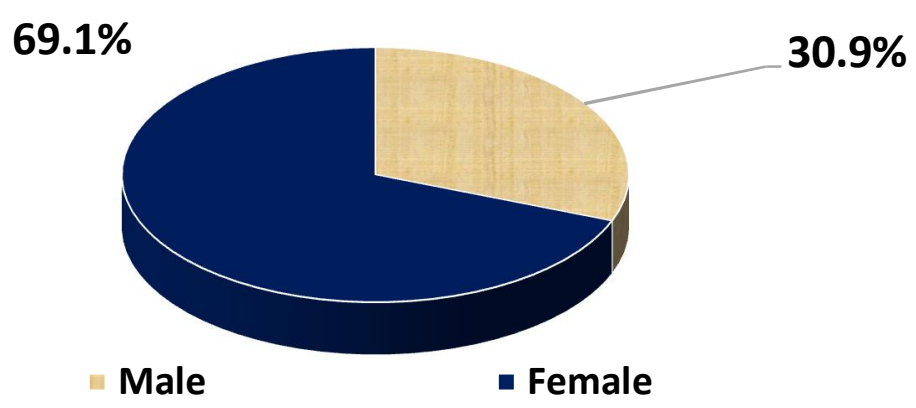

Figure (1): Distribution of the staff nurses according to their gender $(n=337)$.

male.

Figure (1) shows that the more than two thirds (69.1\%) of the staff nurses are female and one third (30.9\%) of them are

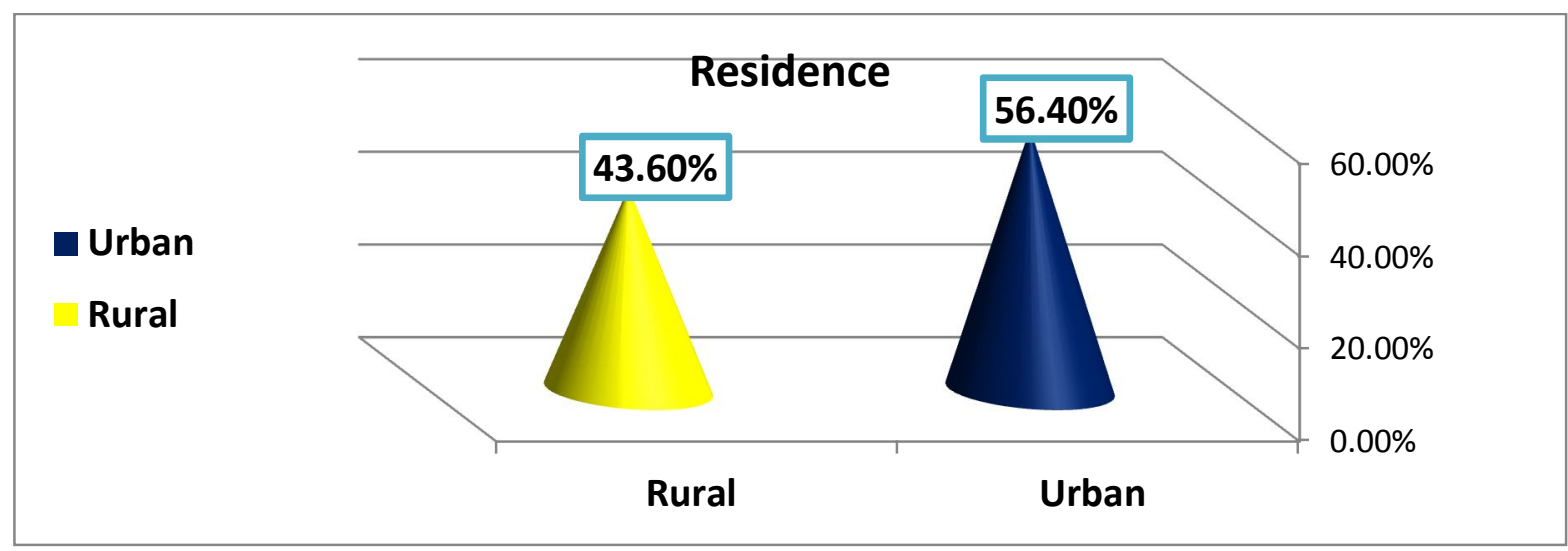

Figure (2): Distribution of the staff nurses according to their residence $(n=337)$. rural area.

Figure (2) shows that there are $(56.4 \%)$ of the staff nurses are living in urban area, and there are $(43.6 \%)$ of them living in

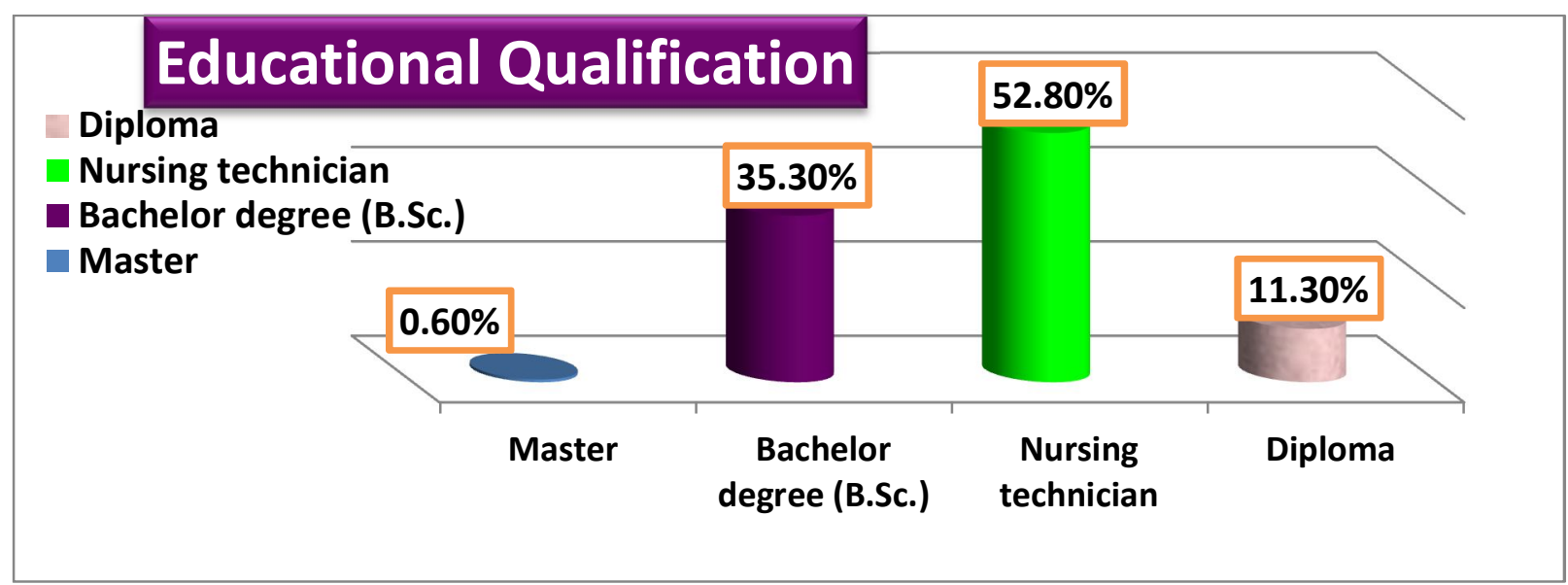

Figure (3): Distribution of the staff nurses according to their educational qualification $(\mathbf{n}=\mathbf{3 3 7})$.

Figure (3) shows that there are (52.8\%) of the staff nurses have Nursing Technician degree, $(35.3 \%)$ of them have Bachelor degree, $(11.3 \%)$ of them have Diploma degree, and there are only $(0.6 \%)$ of them have master degree. 


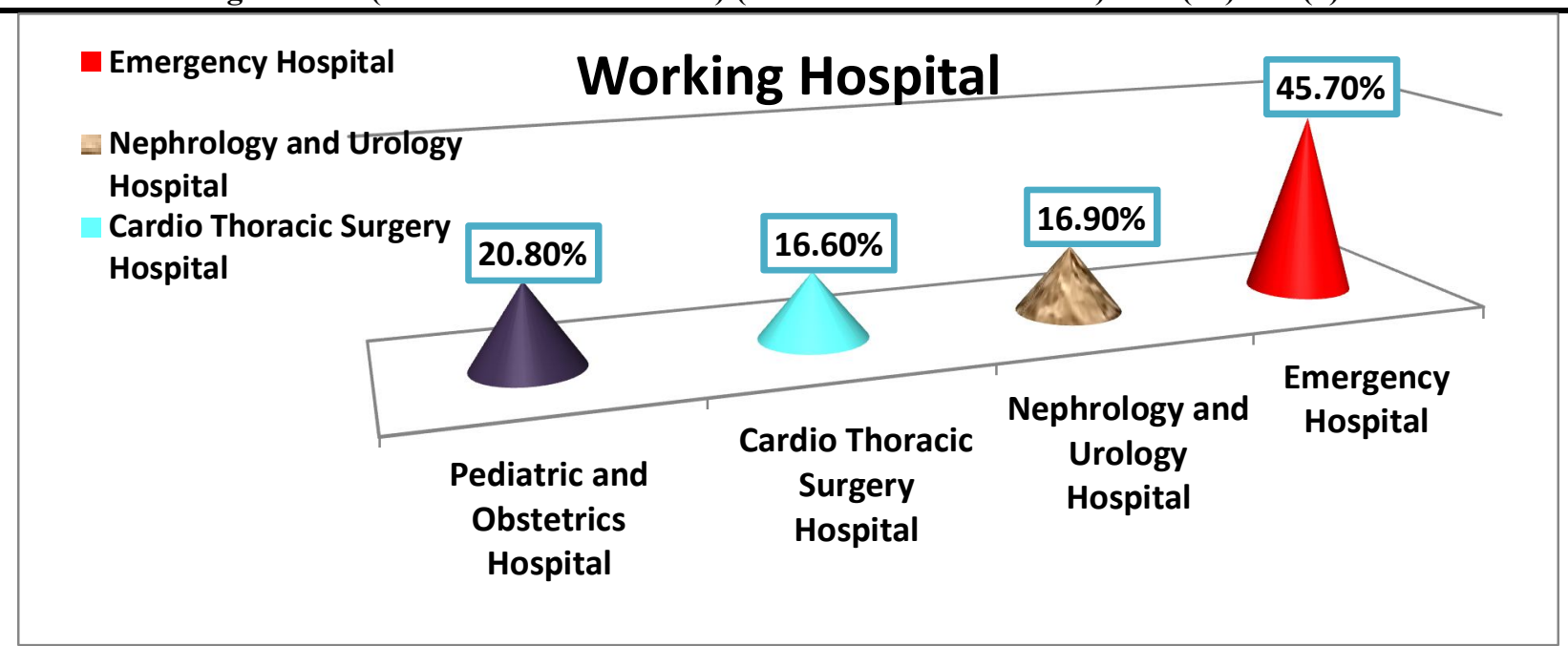

Figure (4): Distribution of the staff nurses according to their working hospitals $(n=337)$.

Figure (4) shows that there are (45.7\%) of the staff nurses work in emergency hospital, while lowest percentage work in nephrology and urology hospital (16.9\%) and cardio thoracic surgery hospital $(16.60 \%)$.

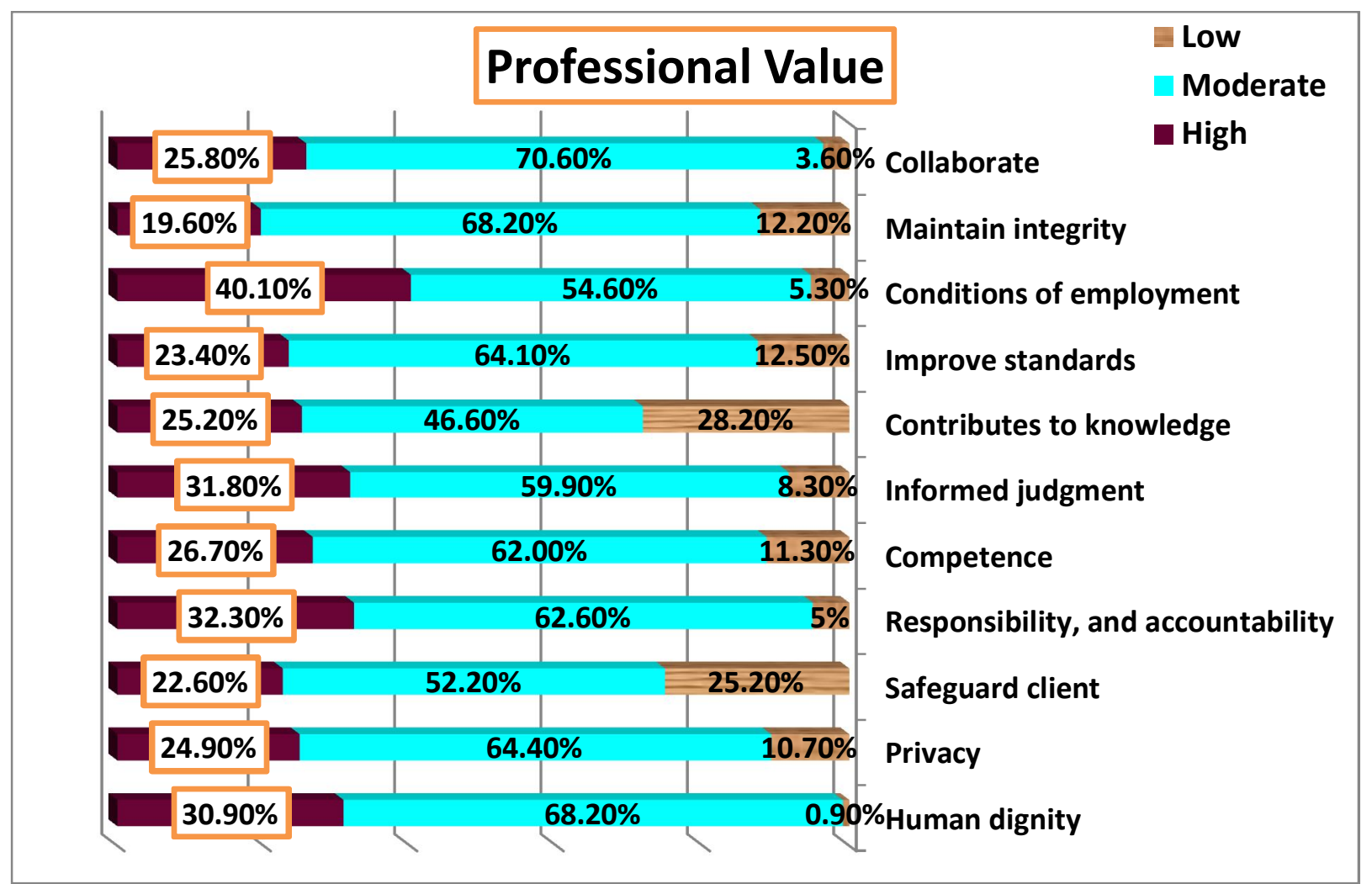

Figure (5) Distribution of Staff Nurses Level of Professional Value subscales $(n=337)$.

Figure (5) presents that the staff nurses have moderate level for all subscales of professional values as; for Human dignity scale there are (68.2\%); for Privacy (64.4\%); for Safeguard client (52.2\%); for Responsibility, and accountability (62.6\%); for Competence (62.0\%); for Informed judgment (59.9\%); for Contributes to knowledge (46.6\%); for Improve standards $(64.1 \%)$; for Conditions of employment (54.6\%); for Maintain integrity $(68.2 \%)$; and for Collaborate $(70.6 \%)$.

Also, it was noted from figure (5) that the subscales which have high percentage of "high level" from staff nurses point of view are: Conditions of employment; Human dignity; Responsibility, and accountability; informed judgment; Competence; and Collaborate (40.1\%. 30.9\%, 32.3\%, 31.8\%, 26.8\%, and 25.8\% respectively). While the subscales which have high percentage of "low level" from staff nurses point of view are Contributes to knowledge and Safeguard client ( $28.2 \%$ and $25.2 \%$ respectively). 


\section{Total Professional Values}

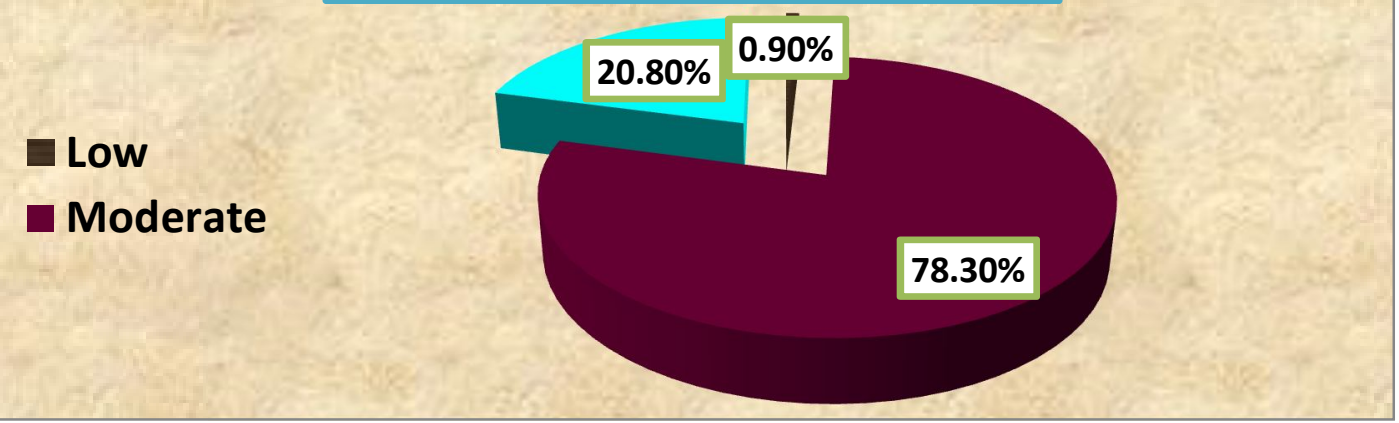

Figure (6): Distribution of the staff nurses according to their total score level of professional value perception $(\mathrm{n}=\mathbf{3 3 7})$.

Figure (6) shows that there are (78.3\%) of the staff nurses have moderate score level of total professional value, $(20.8 \%)$ of them have high score level degree, and there are only $(0.90 \%)$ of them have low score level degree.

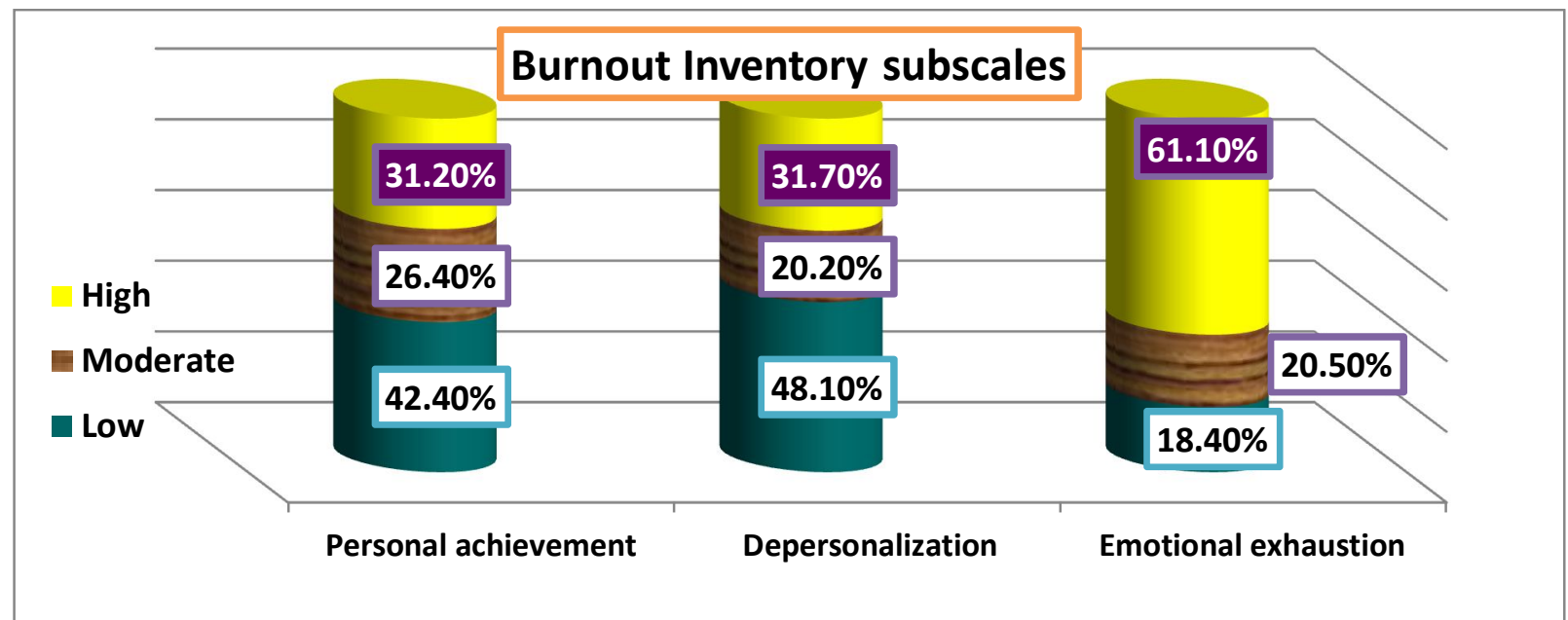

Figure (7): Distribution of the staff nurses according to their score level of burnout inventory subscales $(n=337)$.

Figure (7) presents that near to two thirds (61.1\%) of staff nurses have a high level to emotional exhaustion subscale; and only $(18.40 \%)$ have low score level. Regarding the depersonalization subscale, near to one thirds $(31.7 \%)$ of staff nurses have a high level, and highest percentage (48.10\%) of them have low level score. Speaking about personal achievement, there are (42.4\%) of them have a low level of personal achievement and (3120\%) of them have high level.

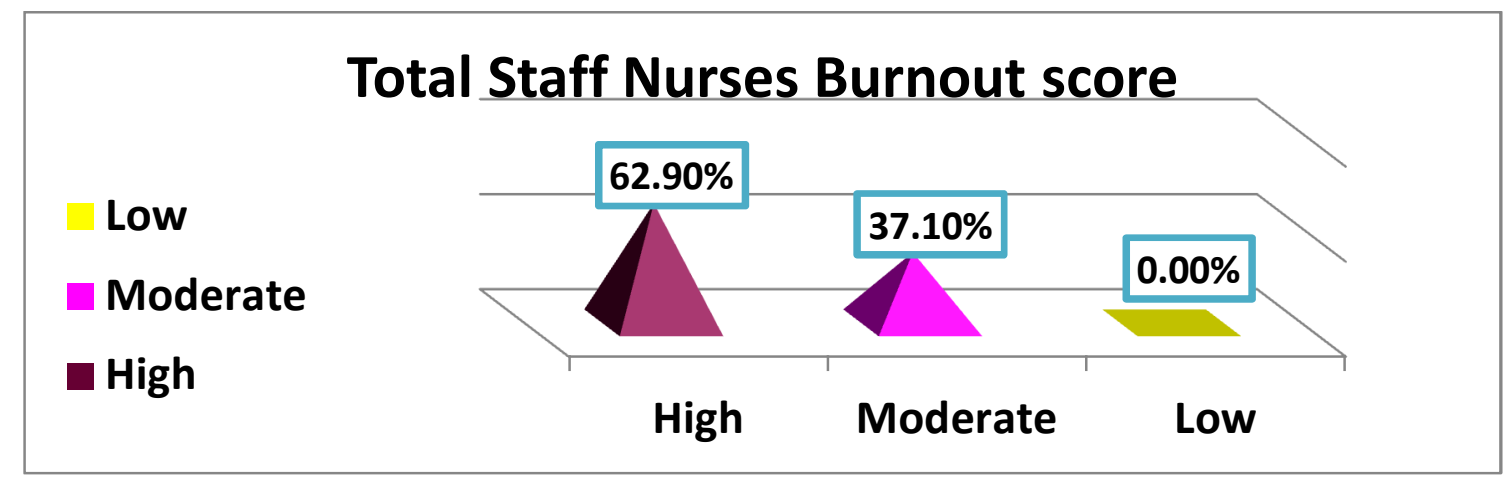

Figure (8): Distribution of staff nurses' perception regarding levels of Maslach's burnout inventory $(n=337)$.

Figure (8) presents that total score level of staff nurses regarding levels of Maslach's burnout inventory; there are (62.90\%) of staff nurses have a high level; and (37.10\%) have moderate score level.

Table (2): Correlations between the burnout of staff nurses' and their professional value $(n=337)$.

\begin{tabular}{|c|c|c|c|c|c|}
\hline Items & & $\begin{array}{l}\text { Emotional } \\
\text { exhaustion }\end{array}$ & Depersonalization & $\begin{array}{c}\text { Personal } \\
\text { achievement }\end{array}$ & $\begin{array}{c}\text { Total Maslach's } \\
\text { Burnout } \\
\text { Inventory }\end{array}$ \\
\hline \multirow{2}{*}{ Human dignity } & $\mathbf{r}$ & 0.019 & -0.221 & 0.250 & -0.222 \\
\hline & $P$-value & 0.730 & $0.0001 * *$ & $0.0001 * *$ & $0.0001 * *$ \\
\hline \multirow[t]{2}{*}{ Privacy } & $\mathbf{r}$ & -0.285 & -0.156 & 0.113 & -0.313 \\
\hline & $P$ - value & $0.0001 * *$ & $0.004 * *$ & $0.038 *$ & $0.0001 * *$ \\
\hline \multirow[t]{2}{*}{ Safeguard client } & $\mathbf{r}$ & 0.040 & -0.377 & 0.140 & -0.261 \\
\hline & $P$ - value & 0.464 & $0.001 * *$ & $0.01 *$ & $0.0001 * *$ \\
\hline Responsibility, and & $\mathbf{r}$ & 0.012 & -0.187 & 0.279 & -0.214 \\
\hline
\end{tabular}


Minia Scientific Nursing Journal (Print - ISSN 2537-012X) (Online - ISSN 2785-9797) Vol. (10) No. (1) December 2021

\begin{tabular}{|c|c|c|c|c|c|}
\hline accountability & $P$ - value & 0.828 & $0.001 * *$ & $0.0001 * *$ & $0.0001 * *$ \\
\hline \multirow[t]{2}{*}{ Competence } & $\mathbf{r}$ & -0.124 & -0.347 & 0.206 & -0.333 \\
\hline & $P$ - value & $0.022 *$ & $0.0001^{* *}$ & $0.0001 * *$ & $0.0001 * *$ \\
\hline \multirow[t]{2}{*}{ Informed judgment } & $\mathbf{r}$ & $0.038-$ & 0.006 & 0.361 & -0.132 \\
\hline & $P$-value & 0.491 & 0.915 & $0.0001 * *$ & $0.015^{*}$ \\
\hline \multirow{2}{*}{ Contributes to knowledge } & $\mathbf{r}$ & 0.038 & -0.453 & 0.156 & -0.302 \\
\hline & $P$-value & 0.485 & $0.0001 * *$ & $0.004 * *$ & $0.0001 * *$ \\
\hline \multirow[t]{2}{*}{ Improve standards } & $\mathbf{r}$ & -0.252 & -0.246 & 0.040 & -0.301 \\
\hline & $P$ - value & $0.0001 * *$ & $0.0001 * *$ & 0.461 & $0.0001 * *$ \\
\hline \multirow{2}{*}{$\begin{array}{l}\text { Conditions } \\
\text { employment }\end{array}$} & $\mathbf{r}$ & -0.177 & 0.050 & 0.159 & -0.211 \\
\hline & $P$-value & $0.001^{* *}$ & 0.357 & $0.003^{* *}$ & $0.0001 * *$ \\
\hline \multirow[t]{2}{*}{ Maintain integrity } & $\mathbf{r}$ & 0.097 & -0.149 & 0.240 & -0.237 \\
\hline & $P$ - value & 0.076 & $0.006^{* *}$ & $0.0001 * *$ & $0.0001 * *$ \\
\hline \multirow[t]{2}{*}{ Collaborate } & $\mathbf{r}$ & -0.180 & -0.277 & 0.238 & -0.352 \\
\hline & $P$ - value & $0.001 * *$ & $0.001 * *$ & $0.001 * *$ & $0.001 * *$ \\
\hline \multirow[t]{2}{*}{ Total professional value } & $\mathbf{r}$ & -0.137 & -0.318 & 0.267 & -0.354 \\
\hline & $P$ - value & $0.012 *$ & $0.001 * *$ & $0.001 * *$ & $0.001 * *$ \\
\hline
\end{tabular}

*Correlation is significant at the 0.05 level $\quad * *$ Correlation is significant at the 0.01 level

Table (2) there are negative associations between emotional exhaustion of the nurse's staff with the subscales of professional values which are (privacy, competence, improve standards, Conditions of employment, and collaborate) with p-values ( $0.0001,0.022$, $0.0001,0.001$, and 0.001 respectively). Regarding correlation between depersonalization with subscales of professional values; there are negative correlations for all subscales of professional values except (informed judgment, and conditions of employment) with pvalues $(0.915$, and 0.357 respectively).

Also, there are positive associations between personal achievement with all subscales of professional values except the subscale (improved standards) with p-value $(=0.461)$

In addition, there are negative associations between total professional values with emotional exhaustion subscale $(\mathrm{r}=-0.137$, $\mathrm{p}=0.012)$ and with depersonalization subscale $(\mathrm{r}=-0.318, \mathrm{p}=0.001)$; while there is positive association between total professional values with personal achievement subscale $(\mathrm{r}=0.267, \mathrm{p}=0.001)$. Also, it was noted from the table that, there is a negative correlational between total professional values of staff nurses and their score of burnout $(r=-0.354, p=0.001)$

\section{Discussion:}

Nurses are affected both mentally and physically at work, and they are particularly vulnerable to job burnout. Nurse burnout can be further differentiated based on clinical duties or specialty. Burnout, commonly defined as a workrelated syndrome characterized by emotional exhaustion, depersonalization, and a sense of reduced accomplishment, negatively affects healthcare team health and productivity, and patient care (Agarwal et al., 2020).

The application of professional values by nurses in their practice is associated with better quality of patient care. They positively impact nurses' judgment and choices in their care for patients and their decisions and actions, complying with standards (Al Shammari et al., 2017; Stainton and Hughson 2017).

In other words, the professional aspect of nursing, including its philosophies, values and ideologies, can be combined with the occupational aspect, and the values derived from this combination should promote the professional satisfaction of individual nurses and the public's recognition of nurses' values. One's professional role in nursing is inseparable from one's ethical values (Shahriari, et al., 2013).

Professional values affect the nurse's quality of life because they determine the motives of someone who is attempting to deal with an ethical dilemma. Professional values are directly related to whether nurse's efforts will result in feeling satisfied and rewarded and play a significant role in the burnout that nurses experience (Kohlen\& McCarthy, 2020).

Nurses with positive professional values tend to be more competent with their employment and achieve greater results than other nurses because of their engagement with, and intense commitment to, the organization. Therefore, nursing values can be understood as a main concept in problem-solving strategies used by the profession to solve current problems (Scoot, 2017). However, investigations that have examined the relationship between nurses' professional values and their burnout level are not rich and there is a need for more studies and researches.

Concerning the distribution of the staff nurses according to their personal data, it was noted from the current study results that there were more than three quarters of staff nurse's aged between $18-<28$ with mean age $26.5 \pm$ 6.1 years; more than half of staff nurses married, and lives in the urban area. Regarding educational qualification and current position; there were more than half of them had a nursing technician, as well as their current position is a nurse.

Also, the current study revealed that majority of staff nurses had from $1-<6$ years with mean experience $5.6 \pm 6.3$ years in the nursing field. In addition there were less than half of the staff nurses work in emergency hospital, while lowest percentage work in nephrology and urology hospital and cardio thoracic surgery hospital.

Concerning the distribution of the staff nurses according to their professional values subscales; it was noted from the current study results that the staff nurses had moderate level for all subscales of professional values as; for Human dignity, Privacy, Safeguard client, Responsibility and accountability, Competence, Informed judgment, Contributes to knowledge, Improve standards, Conditions of employment, Maintain integrity, and for Collaborate.

These results may be as result of nurses' awareness and knowledge about their nursing profession ethics; the study subjects are from different categories of nurses and qualification in which there are high bachelor degree, technical degree, and diploma degree that provide nurses with huge information regarding professional ethics, beliefs and values. 
Also, the study subjects are from different culture and different setting in which enrich them with ethical values and beliefs. They learn from their culture to protect their community setting from unsafe health product and practices as well as their environment may articulated them with values and goals of the nursing profession

This justification come in line with Tehranineshat, et al., (2020) who agreed that the professional values are rooted in personal values that are shaped by an individual's culture, family, environment, religion, and ethnicity. The acquisition of these values is a gradual and evolutionary process and extends throughout one's life. Also, nurses' observance of professional values will not only improve the quality of nursing care, but increase nurses' job satisfaction, professional survival, and organizational commitment (Lin, et al., 2010).

However; these results are not congruent with Allari, et al., (2017) who had a study on 150 Saudi female nurses living in Riyadh and their results show that Saudi female nurses have a high perception of professional values relating to confidentiality, privacy, moral and legal rights, health and safety, and the work environment.

Also, it was noted from the current study results that the subscales which had high level from staff nurses point of view were: Conditions of employment; Human dignity; Responsibility, and accountability; informed judgment; Competence; and Collaborate. While the subscales which had low level from staff nurses point of view were: Contributes to knowledge and Safeguard client.

These results may be due to the nurses recognize their patient right to choose treatment plan; they commitment to follow a written guidelines for practice and care providing; they refuse to participate in care which is ethical opposition to their own professional value; also, they act as a patient advocator. As well as the staff nurses use patient assessment data to develop a nursing care plan; they provide care without judgment to patients lifestyles or values.

This finding in the same line with Bleda et al., (2020) determined whether nursing students attach increasing importance to professional values during the course of their academic degree, concluded that students' scores were higher in the ethics dimension. The two highest scores were for Maintain patient confidentiality for years 1 and 2 (4.77 and 4.68 , respectively) and Safeguard patients' right to privacy for years 3 and 4 (4.95 and 4.98, respectively). Lower scores were observed in the professional expertise dimension across all years, and corresponded to a single item participate in peer review $(3.51,3.38,3.98$, and 3.26, respectively).

Concerning total score of professional values; current study results concluded that there were more than three quarter of the staff nurses had moderate score level of total professional value, less than one quarter of them had high score level degree, and there are only $(0.90 \%)$ of them had low score level degree.

These results are in the same context with the results of Monroe (2017) who had a study to measure the professional values of practicing registered nurses (RNs) in the state of Washington (WA) and revealed from results that nurses have level from moderate to high level mean score of professional values and its subscales as maintain competency in area of practice; accept responsibility and accountability for own practice; and act as a patient advocate

Also, Akbarilakeh et al. (2019) mentioned that near to two-thirds $(58.1 \%)$ of the studied sample had moderate professional vale level and more than one third (36.5\%) of them had high level of professional value.

But Allari (2018) described the perception of professional values and hospital ethical climate among nurses and the correlation between nurses' professional values and hospital ethical climate, revealed that nurses in Jordan have high professional values.

Also, these results are not in line with Sands, (2021) who had a study on 171 Midwestern United States participants from the payor industry and displayed that nurses placed high importance on professional values.

Concerning the distribution of the staff nurses according to burnout subscales score level; current study results presented that there were less than two thirds of staff nurses had a high level to emotional exhaustion subscale; and low percentage of them had low score level. Regarding the depersonalization subscale, near to one thirds of staff nurses had a high level, and near to half as the highest percentage of them had low level score. Speaking about personal achievement, there were less than half of them had a low level of personal achievement and less than one third of them had high level.

These results may be due to staff nurses had job dissatisfaction than head nurses and loss of appreciation due to they were well trained, had high skills which make them over qualified than tasks assigned to them, they work hard and they didn't have the credit for their work, they feel isolated, and they don't had the career progress that as they wanted.

Moreover, this is understandable because nurses deal with deaths daily, go through emotional challenges of losing patients regularly, are constantly faced with consoling grieving relatives of patients, and sometimes have to go on relatively long shifts, thereby causing emotional exhaustion or burnouts

These results are consistent with Stafford and Judd (2010) who investigated the prevalence of psychiatric morbidity and occupational Burnout among Australian gynecologic oncologists in a survey study in Turkey where $42 \%$ had high emotional exhaustion scores, and 20\% had high scores of depersonalizations, and $35.6 \%$ had low scores of personal accomplishment.

Also, this was consistent with Abdo et al. (2015) found that more than half $(52.8 \%)$ of the studied nurses had a high level of emotional exhaustion, nearly half (48.2\%) of them had low depersonalization, and most (96.5\%) of them had reduced in personal accomplishment.

Moreover, Anwar and Elareed (2017) revealed that $54.6 \%$ of nurses had average levels of emotional exhaustion, $48 \%$ scored high on depersonalization, and the majority (77.5\%) had high levels of reduced personal accomplishment.

Also, Sillero and Zabalegui (2018) determined the influence of organizational factors of the perioperative nurse's work environment on the three burnout dimensions: emotional exhaustion, depersonalization, and personal accomplishment in a perioperative care unit at a university hospital in Barcelona, Spain, showed emotional exhaustion in $43 \%$ of nurses, depersonalization in $21 \%$, and reduced personal accomplishment in 53\%

In addition these finding are in the same line with Soltan et al. (2020), nearly one third (30.0\%) of the studied nurses had high emotional exhaustion, depersonalization and nearly half $(45.0 \%)$ of them reduced personal capacity. And Jalili et al. (2021), showed that more than one third and more 
than half of nurses experienced moderate and high levels in the emotional exhaustion axis, respectively,

Almodibeg \& Smith (2021) detected the level of burnout and its most significant causes among perioperative nurses in a regional hospital in Saudi Arabia, showed that a high level of emotional exhaustion was detected in $87.2 \%$. Similarly, a high level of depersonalization was detected in $56.4 \%$, while $15.4 \%$ of nurses showed a low sense of personal accomplishment.

Concerning total score of nurses burnout; current study results concluded that there were less than two third of staff nurses had high total score levels of Maslach's burnout inventory; more than one third of them have moderate score level; and no one of them had low burnout level score.

These results may be due to the staff nurses have low satisfaction level because of their poor work environment that affect the quality of work life which is still need for more improvement; as they mentioned that rotating schedules negatively affect their work and life. Also, they reported that the workload is too heavy, and they need more support from administrative authority's personnel

Also, these results confirmed that nurses had the highest vulnerability in experiencing burnouts. This can be attributed to the studied nurses feeling used up at the end of the workday, fatigued when they get up in the morning, facing another day on the job, and job burnout.

These results confirmed with Sillero and Zabalegui (2018) who determined the influence of organizational factors of the perioperative nurse's work environment on the three burnout dimensions: emotional exhaustion, depersonalization, and personal accomplishment in a perioperative care unit at a university hospital in Barcelona, Spain, reported that $41 \%$ of perioperative nurses in Spain had a moderate or high risk of burnout.

Also, Soltan et al. (2020) studied the prevalence of burnout levels and worked stress among medical oncologists working at multi-centers in Egypt (Cairo, Menoufia, Fayoum, and Assiut university hospitals). Out of 100 participants, 32\% were overall burnout positive as they met the burnout subscales criteria.

As well as, Jalili et al. (2021) described the prevalence of burnout among healthcare professionals and the associated factors in the capital city of Tehran, Iran, mentioned that more than half $(53.0 \%)$ of the studied health care workers experienced high levels of burnout.

González -Pando et al., (2021) analyzed the relationship between professional values, perceived stress, and professional quality of life among nurses during the COVID19 crisis, mentioned that the majority of the studied sample had high level of burnout.

Concerning the correlations between staff nurses professional values and burnout level; the current study show that there were negative associations between emotional exhaustion of the nurse's staff with the subscales of professional values which are (privacy, competence, improve standards, Conditions of employment, and collaborate). Regarding correlation between depersonalization with subscales of professional values; there were negative correlations for all subscales of professional values except (informed judgment, and conditions of employment).

Also, there were positive associations between personal achievement with all subscales of professional values except the subscale (improved standards). In addition, there were negative associations between total professional values with emotional exhaustion subscale and with depersonalization subscale; while there is positive association between total professional values with personal achievement subscale. Also, it was noted from the table that, there is a negative correlational between total professional values of staff nurses and their score of burnout.

This may be because professional values consist of beliefs, attitudes, and feelings that guide nurses' decisions and actions. And the nurses who had high professional value become more responsible, safeguard patient, improve the standard of care and maintain competence in their area of practice and this add stressors for nurses and may lead to burnout.

These correlations are confirmed with Akbarilakeh et al., (2019) shown that there was a significant reverse relationship between professional value score and burnout score.

Also, these findings are in the same line with a study done by Zhou et al. (2020), explored the mediating effect of emotional labor between professional values and job burnout among ICU nurses in Nanchang mentioned that professional values of ICU nurses had a direct predictive impact on job burnout.

However, these results are not in line with Abdalla et al. (2016) results who aimed to find out the relationship between professional values and burnout among nurses in Assuit University hospital in general and specialized medical, surgical and intensive care units, concluded that there was a positive correlation between nurses' professional values and burnout.

\section{Conclusion:}

It can be concluded from the current study that staff nurses had moderate level for all subscale of professional values as; for Human dignity, Privacy, Safeguard client, Responsibility and accountability, Competence, Informed judgment, Contributes to knowledge, Improve standards, Conditions of employment, Maintain integrity, and for Collaborate. Also, current study results concluded that there were more than three quarter of the staff nurses had moderate score level of total professional value, less than one quarter of them had high score level degree.

In addition there were less than two thirds of staff nurses had a high level to emotional exhaustion subscale; and low percentage of them had low score level. Regarding the depersonalization subscale, near to one thirds of staff nurses had a high level, as well there were less than half of them had a low level of personal achievement. Also, there were less than two third of staff nurses had high total score levels of Maslach's burnout inventory; more than one third of them have moderate score level; and no one of them had low burnout level score. Also, it was noted that, there was a negative correlational between total professional values of staff nurses and their score of burnout.

\section{Recommendations}

Based on current study results these recommendations are introduced:

- Design a continuously training program for staff nurses to improve their professional values perception and decrease their burnout level should be done by head nurses.

- Remove all organizational factors that hinder decision making process or generate conflict and create a healthy 
work environment and culture by the hospitals administrators.

- Participate the nurses in the development of hospital policy must be done by administrators to increase nurses creativity and production to reduce their burnout level.

- Create an empowering work environment with a mutual trust and respect for all parts should be done by top level managers.

- Improve nursing work conditions using the different strategies as; reduce nurse workload through appropriate staffing, distribute resources fairly, provide professional development opportunities, and provide effective leadership should be done by the nurse managers.

\section{References}

(1) Abbas, A., Ali, A., Bahgat, S. \& Shouman, W. (2020) Prevalence, associated factors, and consequences of burnout among ICU healthcare workers: an Egyptian experience. The Egyptian Journal of Chest Diseases and Tuberculosis 2019, 68:514-525.

(2) Abdalla, N. A., Yousef, H. R., \& Morsy, S. M. (2016). Professional values and burnout among nurses. Assiut Scientific Nursing Journal, 4(8), 189-194.

(3) Abdalla, N.A., Yousef, H.R., \& Morsy, S,M. (2016). Professional values and Burnout among nurses. Assiut scientific nursing journal; 4 (8): 188- 194.

(4) Abdo, S. A., El-Sallamy, R. M., El-Sherbiny, A. A., \& Kabbash, I. A. (2015). Burnout among physicians and nursing staff working in the emergency hospital of Tanta University, Egypt. East Mediterr Health J, 21(12), 906-915.

(5) Abo Elmagd, N. S., \& Ahmed, M., E. (2019). Factors Affecting Nurses' Career Development and Its' Relation to Job Burnout. Assiut Scientific Nursing Journal, 7(19), 186196.

(6) Agarwal, S. D., Pabo, E., Rozenblum, R., \& Sherritt, K. M. (2020). Professional dissonance and burnout in primary care: a qualitative study. JAMA internal medicine, 180(3), 395-401.

(7) Akbarilakeh, M., Goodarzi, F., \& Oroei, M. (2019). The association between professional value and occupational burnout among nurses working in the emergency department. Social Determinants of Health, 5(1), 36-47.

(8) Al Shammari F., Grande R., Vicencio D., and Al Mutairi S. (2017). Nurses' professional values on patient care provisions and decisions. Journal of Nursing Education and Practice; 7(.9):78-84.

(9) Allari, R. (2018). Hospital ethical climate and it's impact on nurses' professional values. J Nurs Healthc Res, 4, 13-9.

(10) Allari, R., Samantha, I, Mowafa, H. (2017). Professional values among female nursing students in Saudi Arabia. Studies in Health Technology and Informatics, v 238:231234.

(11) Almodibeg, B. A., \& Smith, H. (2021). A cross $\square$ sectional survey to explore the prevalence and causes of occupational burnout syndrome among perioperative nurses in Saudi Arabia. Nursing Open, 8(1), 364-371.

(12) American Nurses Association. (2015). Code of ethics for nurses with interpretive statements (8th ed.). American Nurses Association.

(13) Anwar, M. M., \& Elareed, H. R. (2017). Burnout among Egyptian nurses. Journal of Public Health, 25(6), 693-697.

(14) Ayla, I.A., Ozyazicioglu, N., Atak, M.\& Surenler, S., (2018). Determination of Professional Values in Nursing Students. Internalitional journal of caring science ; 11(1)254 - 257.

(15) Bleda, S., Alvarez, I., \& Prat, M. (2020, June). The perceptions of professional values among students at a
Spanish Nursing School. In Healthcare (Vol. 8, No. 2, p. 74). Multidisciplinary Digital Publishing Institute.

(16) Elsheikh, M., \& Naga, A. E. (2021). Burnout among Egyptian health care workers and its associations. Egyptian Journal of Psychiatry, 42(1), 43.

(17) González-Pando, D., González-Nuevo, C., GonzálezMenéndez, A., Alonso-Pérez, F., \& Cuesta, M. (2021). The role of nurses' professional values during the COVID-19 crisis. Nursing Ethics, 09697330211034250.

(18) Heijden, B.V., Mahoney, C.B., \& Xu, Y. (2019). Impact of job demands and resources on Nurses' burnout and occupational turnover intention towards an age-moderated mediation model for the Nursing profession. Int. J. Environ. Res. Public Health; 16, 2011.

(19) Jalili, M., Niroomand, M., Hadavand, F., Zeinali, K., \& Fotouhi, A. (2021). Burnout among healthcare professionals during COVID-19 pandemic: a crosssectional study. International Archives of Occupational and Environmental Health, 1-8.

(20) Kim, H.L., \& Yoon, S.H. (2018). Effects of Group Rational Emotive Behavior Therapy on the Nurses' Job Stress, Burnout, Job Satisfaction, Organisational Commitment and Turnover Intention. J. Korean Acad. Nurs. , 48, 432-442.

(21) Kim, K., Han, Y., \& Kim, J. (2015). Korean nurses' ethical dilemmas, professional values and professional quality of life. Nurs Ethics: 2015:22(4):467-78.

(22) Kohlen, H. \& McCarty, J. (2020). Nursing Ethics: Feminist Perspectives. E-book, Switzerland: Springer Nature AG.

(23) Lin, Y.H, Wang- Liching, S., Yarbrough, S., Alfred, D., Martin, P. (2010). Changes in Taiwanese nursing student values during the educational experience. Nurs Ethics 2010;17(5):646e54

(24) Long, C.S., Kowang, T.O., Ping, T.A. (2014). Muthuveloo, R. Investigation on the impact of job stressors on nurses in Malaysia. Asian Soc. Sci., 10, 67-77.

(25) Maslach, C. Jackson, S.E., Leiter, M. P., Schaufeli, W.B. \& Schwab, R.L. (1997). The Maslach Burnout Inventory (MBI) and MBI Manual

(26) Monroe, H. (2017). Education and Experience in Nursing Professional Values Development. Doctoral dissertation; Seattle Pacific University; 149P.

(27) Monroe, H. (2019). Nurses' professional values: Influences of experience and ethics education. Journal of Wiley clinical Nursing. 28:2009-2019.

(28) Pérez-Fuentes, M.D.C., Jurado, M.D.M.M., Martínez, A.M., and Linares, J.J.G. (2019). Burnout and Engagement: Personality Profiles in Nursing Professionals. J. Clin. Med., 8, 286.

(29) Poorchangizi, B., Borhani, F., Abbaszadeh, A., Mirzaee M., Farokhzadian, J. (2019). Professional values of nurses and nursing students: a comparative study. BMC Medical Education; 19: 438.

(30) Posluszny L, Hawley DA. (2017). Comparing professional values of sophomore and senior baccalaureate nursing students. J Nurs Educ.; 56(9):546-50.

(31) Sands, K. J. (2021). Professional Values of Corporate Nurses. Doctoral dissertation. College of Health Professions, Walden University; 165P.

(32) Schaufeli, W. B., Leiter, M. P., Maslach, C. \& Jackson, S. E. (1996). The Maslach Burnout Inventory (MBI) and MBI Manual. Manual 4th ed. Mind Garden, Inc.

(33) Scoot, A.P. (2017) Key Concepts and Issues in Nursing Ethics. 1st ed. Switzerland: Springer Nature AG.

(34) Shahriari, M., Mohammadi, E., Abbaszadeh, A., \& Bahrami, M. (2013). Nursing ethical values and definitions: A literature review. Iranian journal of nursing and midwifery research, 18(1), 1. 
(35) Sillero, A., \& Zabalegui, A. (2018). Organizational factors and burnout of perioperative nurses. Clinical Practice and Epidemiology in Mental Health, 14, 132-142.

(36) Soltan, M. R., Soliman, S. S., Al-Hassanin, S. A., ElSherief, W. A., Elnaggar, M. S., \& Gohar, S. F. (2020). Burnout and work stress among medical oncologists: Egyptian multi-centric study. Middle East Current Psychiatry, 27(1), 1-6.

(37) Stafford L, Judd F (2010) Mental health and occupational wellbeing of Australian gynecologic oncologists. Gynecol Oncol 116:526-532 Medline, Google Scholar

(38) Stainton K., and Hughson J. (2017). Tabbner's Nursing Care: Theory and Practice. 7th ed., Australia: Elsevier Health Sciences, pp. $41-45$.

(39) Tehranineshat, B., Torabizadeh C., Bijani, M. (2020). A study of the relationship between professional values and ethical climate and nurses' professional quality of life in Iran. International Journal of Nursing Sciences 7 (2020) 313-319.

(40) World Health Organization. (WHO). (2019). Burnout an "Occupational Phenomenon": International Classification of Diseases. Available online: https://www.who.int/mental_health/evidence/burn-out/en/

(41) Xie, J., Li, J., Wang, S., Li, L., Wang, K., Duan, Y., . . . Cheng, A. S. (2021). Job burnout and its influencing factors among newly graduated nurses: A cross $\square$ sectional study. Journal of Clinical Nursing, 30(3-4), 508-517.

(42) Zhou, S., Yu, C., Zha, L., \& Zhang, S. (2020). Mediating effect of emotional labor between professional values and job burnout among ICU nurses. Chinese Journal of Modern Nursing, 26, 1048-1053. 